\title{
L'utilisation du drapeau dans la communication internationale des entreprises
}

Roger Ourset

\section{(2) OpenEdition}

1 Journals

Édition électronique

URL : http://journals.openedition.org/communicationorganisation/1544

DOI : 10.4000/communicationorganisation. 1544

ISSN : $1775-3546$

Éditeur

Presses universitaires de Bordeaux

Édition imprimée

Date de publication : 1 mai 1992

ISSN : 1168-5549

Référence électronique

Roger Ourset, "L'utilisation du drapeau dans la communication internationale des entreprises », Communication et organisation [En ligne], 1 | 1992, mis en ligne le 26 mars 2012, consulté le 04 mai 2019. URL : http://journals.openedition.org/communicationorganisation/1544; DOI : 10.4000/ communicationorganisation. 1544

Ce document a été généré automatiquement le 4 mai 2019.

(c) Presses universitaires de Bordeaux 


\title{
L'utilisation du drapeau dans la communication internationale des entreprises
}

\author{
Roger Ourset
}

1 Imaginons pendant quelques instants les impressions visuelles pouvant guetter un client du groupe AIR FRANCE. Qu'il consulte la brochure touristique de Jet-Tours ou son titre de transport, qu'il embarque à bord de la navette routière pour se rendre à l'aéroport ou qu'il patiente en observant les jumbos alignés sur le tarmac, il est confronté à une politique de couleur qui s'articule avec insistance autour du tricolore hachuré, logo omniprésent de l'entreprise depuis une dizaine d'années.

Il est partout et impérieux : seule note de couleur sur la blancheur glacée de la couverture des rapports d'activité, déployé fièrement sur l'empennage des appareils, polymorphe au point de devenir pirogue au Sénégal, coquillage en Polynésie, cornemuse en Écosse, allant même jusqu'à s'immiscer dans le plumage de l'hippocampe ailé, son prédécesseur et emblème historique de la Compagnie.

3 Notre propos n'est pas de discuter de la permanence ou de la notoriété de ce logo qui, somme toute, remplit avec brio et quelque excès son rôle de signature. C'est plutôt sur sa composition et le choix de ses couleurs que nous souhaitons attirer l'attention. Sans vouloir atténuer les qualités de son esthétique, force est de reconnaître qu'il ne fait que reprendre le drapeau officiel de la FRANCE. Le plus étonnant dans ce choix est qu'il paraitt aller de soi, dans la mesure où, pour beaucoup, il n'en existerait pas d'autres, en vertu d'un théorème aussi simple que simpliste : à Compagnie Nationale couleurs nationales, exhibées avec d'autant plus de vigueur que l'entreprise doit rayonner à l'échelle du monde entier.

4 L'exemple d'AIR FRANCE illustre avec éclat les relations que la publicité peut entretenir avec la vexillologie. Derrière ce vocable inusité se dissimule l'ensemble des études portant sur les drapeaux et leur symbolique; discipline auxiliaire de l'Histoire, elle joue dans 
l'élaboration de nombreuses politiques de communication internationale un rôle important, quoiqu'anonyme et largement ignoré.

5 Sans en avoir l'exclusivité, le secteur du transport aérien constitue un lieu d'investigation privilégié, notamment dans son activité «passagers ». Le recours à la vexillologie y est devenu tellement habituel qu'il semble en avoir toujours été ainsi et être consubstantiel à ce genre d'activité. Remontons les années et essayons de démonter certaines certitudes.

6 En troquant sa couleur fétiche, le bleu marine, pour le tricolore national, AIR FRANCE a repris, à quelques années d'intervalles, la démarche d'ALITALIA. Faisant à l'époque figure de pionnier, la compagnie italienne créa en effet l'événement en frappant l'empennage de ses appareils d'un A majestueux, stylisé en vert-blanc-rouge. Le ton était donné et rapidement, de nombreuses compagnies allaient modifier radicalement leur identité visuelle. Les oiseaux, flèches et autres symboles des temps héroïques de l'aviation vont être relégués ou tout simplement abandonnés au profit de jeux de couleurs très chatoyantes, mais pour l'essentiel puisés dans le patrimoine vexillologique du pays d'origine. Il est intéressant de rapporter ces modifications à l'époque où elles se sont produites: grâce aux nouveaux longs courriers-gros porteurs, les compagnies connaissaient une très rapide et très large extension spatiale de leurs activités et une forte internationalisation de leur clientèle. La détermination des nouvelles couleurs dominantes de leur communication universelle n'était dont en rien fortuite. Des choix semblables se posent à toute institution au seuil de son développement international. Concernant le transport aérien, la justification d'une telle floraison nationaliste a été vite trouvée et encore plus aisément reçue. De par son actionnariat, souvent majoritairement public, de par ses droits de trafic octroyés par autorisation ministérielle, une compagnie aérienne est facilement perçue comme un instrument de présence et de fierté nationales. Dès lors elle peut utiliser sans vergogne les symboles du pays qu'elle aurait ainsi plus particulièrement vocation à représenter. Dans le monde anglo-saxon, on la nomme d'ailleurs souvent flag-carrier et c'est vraisemblablement dans cet esprit qu'AIR FRANCE avait conçu son slogan : The Best of France to the World.

7 L'exemple le plus achevé de cette approche de communication internationale fut donné par la compagnie britannique BRITISH AIRWAYS, fruit de la fusion BEA/BOAC : l'oiseausymbole est exilé à une très modeste place à l'avant de l'appareil afin de laisser l'empennage arborer un graphisme représentant explicitement une portion de l'Union Jack. Plus tard, la dénomination commerciale de l'entreprise sera même réduite au seul mot « BRITISH » que l'on retrouvera partout jusqu'au fond des tasses à café.

8 N'est-il pas paradoxal, voire dangereux, d'opter pour une identité au nationalisme si appuyé alors qu'elle doit soutenir des ambitions commerciales à l'échelle de la planète?

9 Un vexillologue rappellerait à bon escient qu'à travers l'histoire le thème commun des drapeaux représente la victoire d'un groupe d'hommes sur un autre et que l'ancêtre du mât est bien cette pique guerrière au sommet de laquelle était accroché un crâne d'animal ou même d'homme, comme en témoigne encore le drapeau corse et sa saisissante tête de Maure... Peut-être faudrait-il manipuler avec davantage de discernement et de subtilité une symbolique aux effets puissants, même s'ils ne se manifestent le plus souvent que de manière diffuse ou inconsciente.

10 L'exigence des marchés étrangers contraint souvent l'entreprise à modifier les termes de son offre. Entre la recherche d'un degré élevé de standardisation et le souci d'une parfaite adaptation aux caractéristiques locales, le marketing international doit s'attacher à 
définir une stratégie adaptée aux débouchés, moyens et contraintes de l'entreprise. Le choix est délicat, tout comme le climat psychologique qui l'accompagne: l'hésitation, bien que légitime, doit éviter d'apparaître comme de l'indécision. Se manifeste alors fréquemment un désir aigu de sécurisation, qui passe souvent par la mise en évidence des critères d'appartenance au pays d'origine. Ainsi, lors de la rénovation ou de la création de logo, rendue nécessaire par l'obligation d'offrir aux clients étrangers un moyen d'identification clair et mémorisable, surgit une intempérance de couleurs nationales, comme peut s'en apercevoir le visiteur attentif de tout salon international.

11 Le besoin d'authentification est compréhensible, mais l'affirmer avec un tel accent nationaliste n'apparaît guère pertinent, voire banal et rudimentaire, sur des marchés régis par des modes de concurrence s'exprimant davantage en terme de prix, qualité ou délais de livraison. Qu'apporte au poulet congelé français son enrubannement tricolore, alors que le produit négocié au plan mondial est globalement indifférencié et son origine presque anecdotique? Au-delà de leur pauvreté d'analyse et de sensibilité en terme de marketing international, ces réponses traduisent un ethnocentrisme passablement suranné dans une économie fortement interpénétrée. Elles peuvent d'ailleurs réserver à leurs promoteurs de dangereux effets de retour, ainsi que l'ont expérimenté les BRITISH AIRWAYS dans plusieurs parties de l'ancien Empire Britannique. À la longue, leur nationalisme affiché a froissé de larges secteurs de la clientèle en Inde, au Canada ou en Australie, farouchement attachés à leurs propres notions d'indépendance et de souveraineté. L'agacement de ces marchés à fort trafic ethnique a pesé d'un grand poids lors de la définition du cahier des charges imposé au Cabinet LANDOR, retenu pour concevoir la nouvelle identité visuelle de la Compagnie: affirmer les éléments indisciblement britanniques tout en atténuant ceux qui l'étaient agressivement. Le résultat est intéressant à observer : le blanc cède la place au gris perle, le rouge est réduit à un mince filet (traduisant le modernisme : filet laser) et la portion d'Union Jack stylisé est enfouie au cœur d'un blason héraldique, mais dans des tons feutrés, à l'image de la gentry au cosmopolitisme discret. A la nouvelle devise «To Fly To Serve» répond la signature à vocation universelle " The World's Favorite Airline ».

Dans le cas inverse, lorsque l'entreprise choisit de privilégier l'adaptation aux différentes attentes des marchés étrangers, la vexillologie peut aussi jouer un rôle important, mais alors les responsables marketing doivent produire un gros effort d'étude et de tact afin de ne pas choquer en utilisant avec maladresse des symboles qui leur sont peu familiers. Dans le seul domaine des drapeaux, la prudence est de rigueur, tant le Droit ou le protocole des différents pays ont défini de multiples manières d'utiliser les pavillons. Ainsi, dans deux pays proches, le DANEMARK et la FINLANDE, les situations sont diamétralement opposées: alors que le Dannebrog, fanion historique danois, est constitutionnellement la propriété de tout citoyen - explication de sa très forte présence commerciale - l'usage du drapeau finlandais est codifié de façon très rigoureuse.

13 Afin de contourner ces difficultés, tout en ménageant à la vexillologie un rôle important, certaines entreprises choisissent une solution intermédiaire qui permet de banaliser le produit dans chaque pays tout en parvenant à une standardisation élevée. Jusqu'à ces dernières années, ce fut le choix retenu par le groupe d'électronique japonais MATSUSHITA lorsqu'il introduisit sur le marché international la marque NATIONAL. Le nom choisit révélait déjà une stratégie de naturalisation. Mais lui était aussi adjoint un logo, composé d'une lettre $\mathrm{N}$ noir sur fond blanc, encadrée par deux bandeaux rouge et bleu. L'emblème de la marque ressortait ainsi sur champ rouge-blanc-bleu, qui constitue 
le tryptique de couleurs commun à la plupart des pays anglo-saxons du monde développé, et, vu à l'envers, celui de la FRANCE et de nombreux pays, notamment latino-américains, qui s'en sont inspirés. C'est une habile utilisation de certains legs de l'Histoire, qui permet au consommateur d'adopter et d'assimiler facilement à sa culture des produits importés.

Les quelques exemples cités montrent les possibilités et les pièges qu'offre la vexillologie aux responsables d'une politique de communication internationale. Quels enseignements peut-on retirer d'une observation globale de ses différentes applications?

Un premier constat s'impose : selon leur pays d'origine, les entreprises ne bénéficient pas de l'égalités des chances. D'une part figurent celles issues de pays dont les symboles nationaux sont connus et appréciés : pour SWISSAIR, quoi de plus efficace que la croix blanche sur fond rouge, symbole protecteur du refuge helvétique depuis 1339 et inspirateur du pavillon de la Croix-Rouge unanimement respectée. D'autre part se situent les entreprises pouvant difficilement miser sur les symboles de leur patrie, quand ceux-ci sont à peu près inconnus ou liés à de bien désagréables souvenirs.

Dans le premier sous-groupe se pressent les entreprises originaires du Tiers-Monde. À de très rares exceptions - ETHIOPIAN AIRLINES et ses couleurs rasta vert-jaune-rouge, la vexillologie y joue un rôle mineur lorsque s'internationalise fortement une entreprise qui en est issue. À observer la sobre livrée bleue et jaune des avions des SINGAPORE AIRLINES, personne ne soupçonne le drapeau blanc et rouge frappé du croissant et des cinq étoiles de la Ville-Etat.

Le second sous-groupe est constitué par les entreprises ayant compris la charge émotionnelle négative véhiculée par l'évocation des symboles de leur pays. Ainsi, depuis plus de 30 ans sur les marchés extérieurs, les entreprises allemandes jouent avec beaucoup de finesse sur un registre nécessitant un extrême doigté: en dépit de l'exception qu'a présenté le drapeau du régime nazi dans la continuité historique des couleurs nationales allemandes, redevenues officielles avec le retour de la démocratie, très peu de firmes allemandes utilisent actuellement le traditionnel noir-rouge-or dans leur communication internationale.

18 Un deuxième point est à évoquer. Une entreprise doit surveiller étroitement l'évolution de la réputation ou du capital - sympathie de son pays d'origine si elle souhaite fonder sur eux une partie de l'identité qu'elle propose à l'étranger. L'exemple NATIONAL en constitue une bonne illustration ; l'actuel logo est toujours bâti autour d'une lettre $\mathrm{N}$ sur fond blanc, mais cette dernière est désormais rouge et contenue dans un cercle de même couleur... Les modifications qui affectent actuellement les PHILIPPINE AIR-LINES sont tout aussi parlantes: profitant de la popularité de Mme AQUINO, la dénomination officielle de la Compagnie est réduite au seul nom PHILIPPINE et un soleil rayonnant jaune vif - couleur de la Présidenta - illumine désormais l'empennage, qui reçoit ainsi une version à peine retouchée du drapeau national.

Une gestion vexillologique de la politique de communication internationale se révèle ainsi plus délicate et complexe que la simple adjonction d'un ruban coloré autour d'un produit ou d'une estampille authentifiant son origine. Elle possède comme avantage de rendre une offre plus chatoyante et plus typée. À l'inverse, elle risque de plonger le produit dans le ghetto du folklore et parfois du grotesque. Le débat n'est pas tranché, d'autant plus qu'il n'est jamais clairement posé. Néanmoins il existe : dans un domaine comme celui de la technologie, il serait intéressant de demander à l'entreprise CAP GEMINI SOGETI, qui opte volontairement pour une identité déliée de toute référence à 
son pays d'origine, ce qu'elle pense de l'impact aux États-Unis de la communication de KIS, qui s'appuyait fortement sur le concept grivois du « French Kiss »...

Le drapeau est à coup sûr un acteur présent dans l'échange international de produits et de services. Utilisé avec humour et diplomatie, il peut devenir un moyen efficace de communication internationale, comme semblaient en convenir dans un récent passé les enfants et les adolescents de l'univers pacifique et fraternel des United Colors of BENETTON...

\section{RÉSUMÉS}

Le recours aux ressources de la vexillologie. études des drapeaux est de leur symbolique, pour promouvoir des produits sur le marché international doit être fait avec prudence car l'usage du drapeau peut connoter un nationalisme pas toujours admis.

\section{AUTEUR}

\section{ROGER OURSET}

Roger Ourset est enseignant et chercheur au groupe ESCP, il y dirige le centre de formation permanente. Il est l'un des rares spécialistes d'une discipline peu connue, la vexillologie. 